\title{
AOR
}

Selected Papers of \#AoIR2019:

The $20^{\text {th }}$ Annual Conference of the Association of Internet Researchers Brisbane, Australia / 2-5 October 2019

\section{CONVENIENCE TRUMPS ALGORITHMS: PREDICTING CONTINUED INTENTION TO USE MUSIC STREAMING SERVICES}

Marika Lüders

Department of Media and Communication, University of Oslo, Norway

A decade after the launch of Spotify, music streaming services (MSS) have become a default way of listening to music. Their offerings to music-listeners now extend beyond abundance and availability to recommendations and curation capabilities. This paper addresses the extent to which music-listeners use the different mechanics of MSS and combines theoretical approaches from technology acceptance studies, collecting/sensemaking in MSS and algorithmic culture/individuation. This theoretical framework is chosen as each approach points to constructs that capture at least part of the use-value of these services, yet that have not been examined in combination.

Empirically, this paper builds on a mixed methods approach combining interviews and a survey on the use of MSS in Norway. While the results from the survey identify what factors matter more for continued intention to use these services, the interviews elucidate how different experiences covary with different practices. Accordingly, I pose the following overall research question: How do patterns of using MSS shape the experienced value of these services?

\section{Theoretical framework and research model}

Studies of technology acceptance and use are common in information systems research (Venkatesh et al., 2012) and have also been applied for understanding antecedents predicting use of streaming services (Hampton-Sosa, 2017; Oyedele \& Simpson, 2018). From these studies, two constructs are included in this study: convenience value and monetary value, where the former reflects the rhetoric of "anything anytime anywhere". Yet, these studies are limited by not addressing usevalues such as features for organizing music, and what importance personalized recommendations play.

Lüders, Marika. (2019, October 03-05). Convenience trumps algorithms: predicting continued intention to use music streaming services. Paper presented at AoIR 2019: The $20^{\text {th }}$ Annual Conference of the Association of Internet Researchers. Brisbane, Australia: AoIR. Retrieved from http://spir.aoir.org. 
While MSS offer vast catalogues of music, people remain attached to their musical preferences and history. The immaterial access-based way of listening to music does not negate the seemingly inherent human desire to collect (Marshall, 2014); and people still experience a need for establishing a sense of psychological ownership, for example by creating playlists (Hagen, 2015; Sinclair \& Tinson, 2017). Few if any studies have tested the importance of such practices. In this study, collecting and sense of ownership is conceptualized as "Will to archive".

Services such as Spotify, Apple Music and Deezer attempt to distinguish themselves by offering the most accurate and relevant personalized recommendations (Morris \& Powers, 2015; Prey, 2018). Communication research abounds with critical discussions of algorithmic culture and algorithmic individuation (Kitchin, 2017; Prey, 2018), yet few studies have examined the perceived value of such recommendations from the perspective of music listeners.

Finally, the study suggests that age predicts continued behavioural intention negatively. Figure 1 presents the resultant hypotheses of this study.

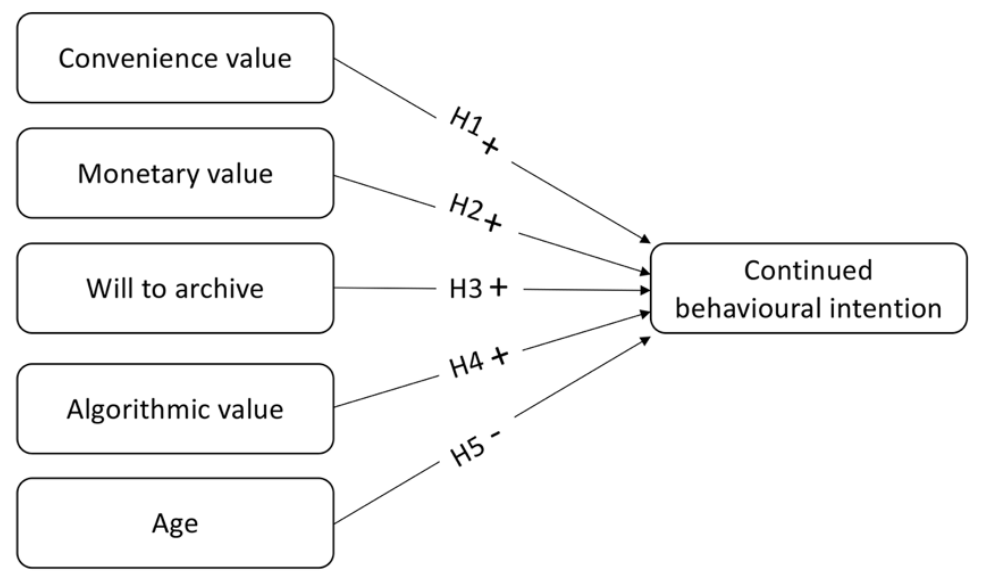

Figure 1: Research model with hypotheses

\section{Method and measurements}

This study is based on interviews with 18 users of streaming services (2017/2018) and an online survey (2018). The interviews were used to develop items for the survey and to explicate findings. The sample for the survey is representative of the Norwegian population and included 1511 respondents, yet only respondents who had single- or multi-subscription accounts (i.e. respondents who pay for MSS) were included for testing the hypotheses $(\mathrm{N}=793)$. For this sub-sample mean age was $39(\mathrm{SD}=14.6)$ and $52 \%$ were male.

Respondents indicated whether they agreed with statements on a 5-point Likert-scale ranging from 1 (completely disagree) to 5 (completely agree). The items were subjected to a principal component analysis with oblimin rotation. Factors that loaded with an eigenvalue of 1 or greater and had at least three loadings using a 60-40 rule were retained. Items that loaded on each factor were summed and averaged. The resultant factors explained $66.7 \%$ of the total variance after rotation. 
Convenience value was measured with four items based on Oyedele \& Simpson (2018) $(\alpha=.87 ; M=4.3 ; \mathrm{SD}=.57)$. Monetary value was measured by adapting three items from Venkatesh et al. (2012) ( $\alpha=.92 ; M=4.0 ; S D=.84)$. Will to Archive is an invented construct based on the qualitative data and was measured with four items $(\alpha=.75 ; M=3.5$;

$\mathrm{SD}=.78$ ). Items included the importance of saving music to link music to a certain time or place, to make it easy to find music for different situation, to avoid losing track of music that matters, and to create a sense of ownership to music. Algorithmic value is an invented construct based on the qualitative data and was measured with four items $(\alpha=.80 ; M=3.5 ; S D=.71)$. Items included whether personalized recommendations included music respondents like, help respondent discover new music, include music respondents end up saving, and help respondent expand their taste in music. Continued behavioural intention was measured by adapting three items from Venkatesh et al. (2012) $(\alpha=.86 ; M=4.0 ; S D=.77)$.

\section{Results and findings}

Correlation coefficients were below the recommended threshold of .7, suggesting multicollinearity is not an issue (Table 1). Hierarchical regression analyses were used to test the hypotheses (Table 2), with the established constructs entered in step 1 and the invented constructs and age entered in step 2.

Table 1: Correlation matrix

\begin{tabular}{lccccc}
\hline & CV & MV & WtA & AV & Age \\
\hline Convenience value: CV & 1 & & & & \\
Monetary value: MV & $.52^{* *}$ & 1 & & & \\
Will to archive: WtA & $.32^{* *}$ & $.26^{* *}$ & 1 & & \\
Algorithmic value: AV & $.37^{* *}$ & $.31^{* *}$ & $.41^{* *}$ & 1 & \\
Age & $-.19^{* *}$ & $-.17^{* *}$ & $-.21^{* *}$ & $-.12^{* *}$ & 1 \\
Continued behavioural intention: $\mathrm{CBI}$ & $.55^{* *}$ & $.52^{* *}$ & $.43^{* *}$ & $.33^{* *}$ & $-.34^{* *}$ \\
\hline
\end{tabular}

Note: ${ }^{* *}$ Correlation is significant at the .01 level (two-tailed).

Table 2: Results from the hierarchical regression analysis

Dependent variable: Continued behavioural intention

\begin{tabular}{lccc} 
& $\boldsymbol{\beta}$ & $\boldsymbol{p}$ & $\boldsymbol{H}$ supported? \\
\hline Step 1 & & & \\
CV & $.34^{* *}$ & .001 & \\
MV & $.37^{* *}$ & .001 & \\
\hline Step 2 & & & \\
CV & $.25^{\star *}$ & .001 & Yes \\
MV & $.33^{* *}$ & .001 & Yes \\
WtA & $.19^{\star *}$ & .001 & No \\
AV & .04 & .202 & Yes \\
Age & $-.19^{* *}$ & .001 & \\
\hline
\end{tabular}

Note: $R^{2}=.38$ for step $1(p<.001) ; \Delta R^{2}=.088$ for step $2(p<.001)$.

In model 1 , convenience value and monetary value account for $38 \%$ of the variation in continued behavioural intention $\left(R^{2}=.38\right)$. In model $2, R^{2}$ increases by $8.8 \%$, making $R^{2}$ 
.46 with a significant F-statistic of $33.15(p<.001)$. This increase is accounted for by will to archive and age.

The results support all hypotheses except $\mathrm{H} 4$ : whereas algorithmic value correlates significantly with all other constructs (Table 1), it does not predict behavioural intention. Perceived monetary value is the strongest predictor followed by convenience value and will to archive. As expected, age is negatively associated with continued behavioural intention.

The analysis of the interviews enquires why and how participants value music streaming services and in particular addresses convenience value, archiving practices and whether or not or how algorithmic recommendations matter. Findings suggest that accessing music anytime and anywhere is the default motivation for using and continuing to use MSS. Yet the abundance of music invokes the question of 'what to listen to?' This problem and/or freedom of abundance is tackled by archiving and relying on algorithmic recommendations. With regard to archiving, people who invest efforts in organizing and curating their own music libraries create added value to their serviceexperience. These personal archives, whether in the form of current lists of favourites or meticulously curated collections of music, come across as safe retreats: musical spaces to rely on for 'my music'. Finally, whereas the survey did not fond algorithmic value to predict continued intention to use MSS, the interviews provide more nuanced accounts and point to how the extent to which personalised recommendations are perceived to matter relates to perceptions of catering for algorithms. Conversely casual listeners pay little attention to recommendations, or perhaps more accurately, do not recognize or see these as recommendations.

\section{Conclusion}

While branding of streaming services has shifted from emphasizing convenience value to e.g. personalized recommendations (Morris \& Powers, 2015), this study indicates that the convenience of accessing any music anytime and anywhere remains crucial for music-listeners. MSS providers may regard it as a central objective to improve how content finds people. It may not matter for the service provider if most people do not grasp how these recommendations work. Yet my attempt to unpack how people use, value and make sense of MSS delineates how the streaming-experience is co-created by music-listeners. Invested listeners get plentiful in return: they cater for and control their service-experience, yet not without simultaneously catering for the provider.

\section{References}

Hagen, A. N. (2015). The Playlist Experience: Personal Playlists in Music Streaming Services. Popular Music and Society 38(5): 625-645.

Hampton-Sosa, W. (2017). The impact of creativity and community facilitation on music streaming adoption and digital piracy. Computers in Human Behavior, 69, 444-453.

Kitchin, R. (2017). Thinking critically about and researching algorithms. Information, Communication \& Society, 20(1), 14-29. 
Marshall, L. (2014). W(h)ither Now? Music Collecting in the Age of the Cloud. In Marshall L, Laing D and Frith S (eds) Popular music matters: essays in honour of Simon Frith. Farnham: Ashgate, pp. 61-72.

Morris, J. W., \& Powers, D. (2015). Control, curation and musical experience in streaming music services. Creative Industries Journal, 8(2), 106-122.

Oyedele, A., \& Simpson, P. M. (2018). Streaming apps: What consumers value. Journal of Retailing and Consumer Services, 41, 296-304.

Prey, R. (2018). Nothing personal: algorithmic individuation on music streaming platforms. Media, Culture \& Society, 40(7), 1086-1100.

Sinclair, G., \& Tinson, J. (2017). Psychological ownership and music streaming consumption. Journal of Business Research, 71, 1-9.

Venkatesh, V., Thong, J. Y., \& Xu, X. (2012). Consumer acceptance and use of information technology: extending the unified theory of acceptance and use of technology. MIS quarterly, 36(1), 157-178. 